Proc. Indian Acad. Sci. (Earth Planet. Sci.), Vol. 101, No. 3, September 1992, pp. 269-282.

(C) Printed in India.

\title{
Static deformation of two welded half-spaces due to dip-slip faulting
}

\author{
SUNITA RANI and SARVA JIT SINGH \\ Department of Mathematics, Maharshi Dayanand University, Rohtak 124001, India
}

MS received 7 January 1992; revised 22 May 1992

\begin{abstract}
Closed-form expressions for the displacements and stresses at any point of either of two elastic half-spaces in welded contact caused by a dip-slip line source obtained earlier are integrated analytically to derive the elastic residual field due to a long dip-slip fault of finite width. The results are valid for an arbitrary dip of the fault. The variation of the displacement field with the distance from the fault as well as with the distance from the interface is studied numerically. It is found that the displacement field is heavily dependent on the dip angle. Contour maps showing the residual elastic field in the two half-spaces caused by a vertical dip-slip fault located in one of the half-spaces are also obtained.
\end{abstract}

Keywords. Dip-slip faulting; long fault; static deformation; welded half-spaces.

\section{Introduction}

The elastic residual field of a long strike-slip fault in a layered half-space has been calculated by Rybicki (1971, 1973), Chinnery and Jovanovich (1972) and others. Freund and Barnett (1976) gave a two-dimensional analysis of surface deformation due to dip-slip faulting in a uniform half-space, using the theory of analytic functions of a complex variable. Rani et al (1991) obtained the elastic residual field in a uniform half-space due to various two-dimensional sources, using the Airy function approach. Singh et al (1992) obtained closed-form analytic expressions for the Airy stress function, displacements and stresses at any point of either of two homogeneous, isotropic, perfectly elastic half-spaces in welded contact due to various two-dimensional sources. Beginning with the expressions for the Airy stress function for a dip-slip line source given by Singh et al (1992), analytic integration over the width of the fault yields the Airy stress function for a long dip-slip fault of arbitrary dip placed in a half-space in welded contact with another half-space. The expressions for the displacements and stresses follow immediately. These analytic closed-form expressions are used to study the variation of the displacement field with the distance from the fault and with the distance from the interface. Contour maps showing the residual displacement and stress field in the two half-spaces due to a vertical dip-slip fault located in one of the half-spaces are also obtained. The modelling of the crustal deformation field should be done with a model consisting of a layer (representing the lithosphere) overlying a viscoelastic half-space (representing the asthenosphere). However, analytic closed-form solution for a dip-slip line source in such a model does not seem feasible. We have, therefore, considered the problem of a dip-slip dislocation in a model consisting of two elastic half-spaces in welded contact. This model brings into focus the effect of a structural discontinuity, but ignores the effect of the free surface. The viscoelasticity 
of the asthenosphere can be taken into consideration by an application of the correspondence principle of viscoelasticity (Singh and Rosenman 1974; Singh and Singh 1990).

\section{Theory}

Let the Cartesian co-ordinates be denoted by $\left(x_{1}, x_{2}, x_{3}\right) \equiv(x, y, z)$, with the $x_{3}$-axis vertically downwards. Consider two homogeneous, isotropic, perfectly elastic halfspaces that are welded along the plane $x_{3}=0$. The upper half-space $\left(x_{3}<0\right)$ is called medium I and the lower half-space $\left(x_{3}>0\right)$ is called medium II, with elastic constants $\lambda_{1}, \mu_{1}$ and $\lambda_{2}, \mu_{2}$, respectively. In the following, the superscript (1) denotes quantities related to medium I and the superscript (2) denotes those related to medium II.

Consider a plane strain problem parallel to the $x_{2} x_{3}$-plane so that

$$
u_{1} \equiv 0, \quad \partial / \partial x_{1} \equiv 0
$$

We define the Airy stress function $U$ (Sokolnikoff 1956) through the relations

$$
\begin{aligned}
& p_{22}=U_{, 33}, \quad p_{23}=-U_{, 23}, \quad p_{33}=U_{, 22}, \\
& \nabla^{2} \nabla^{2} U=0,
\end{aligned}
$$

where $p_{i j}$ are the components of stress and $U_{, 33}=\partial^{2} U / \partial x_{3}^{2}$, etc.

As shown by Singh et al (1992), the expressions for the Airy stress function in the two half-spaces due to a dip-slip line source parallel to the $x_{1}$-axis and passing through the point $\left(y_{2}, y_{3}\right)$ in the lower half-space (medium II) are:

$$
\begin{aligned}
U^{(1)}= & \left(\alpha_{2} \mu_{2} b d s / \pi\right)\left[\operatorname { c o s } 2 \delta \left\{\frac{C_{1}+D_{1}}{2} \tan ^{-1}\left(\frac{x_{2}-y_{2}}{y_{3}-x_{3}}\right)\right.\right. \\
& \left.-\frac{\left(D_{1} y_{3}+C_{1} x_{3}\right)\left(x_{2}-y_{2}\right)}{R^{2}}\right\}+\sin 2 \delta\left\{\frac{\left(C_{1}+D_{1}\right)}{2} \ln R\right. \\
& \left.\left.+\frac{\left(D_{1} y_{3}+C_{1} x_{3}\right)\left(y_{3}-x_{3}\right)}{R^{2}}\right\}\right], \\
U^{(2)}= & \left(\alpha_{2} \mu_{2} b d s / \pi\right)\left[\operatorname { c o s } 2 \delta \left\{\frac{\left(x_{2}-y_{2}\right)\left(x_{3}-y_{3}\right)}{R^{2}}-D_{2} \tan ^{-1}\left(\frac{x_{2}-y_{2}}{x_{3}+y_{3}}\right)\right.\right. \\
& \left.+\frac{C_{2}\left(x_{2}-y_{2}\right)\left(x_{3}-y_{3}\right)}{S^{2}}-\frac{4 C_{2} x_{3} y_{3}\left(x_{2}-y_{2}\right)\left(x_{3}+y_{3}\right)}{S^{4}}\right\} \\
& +\sin 2 \delta\left\{\frac{\left(x_{3}-y_{3}\right)^{2}}{R^{2}}-D_{2} \ln S-\frac{C_{2}\left(x_{3}^{2}-y_{3}^{2}+2 x_{3} y_{3}\right)}{S^{2}}\right. \\
& \left.\left.+\frac{4 C_{2} y_{3} x_{3}\left(x_{3}+y_{3}\right)^{2}}{S^{4}}\right\}\right]
\end{aligned}
$$

where

$$
\begin{aligned}
b & =\text { displacement discontinuity (slip), } \\
d s & =\text { width of the line source, }
\end{aligned}
$$




$$
\begin{aligned}
\delta & =\text { dip angle (figure } 1) \\
\left(x_{2}, x_{3}\right) & =\text { receiver location, } \\
R^{2} & =\left(x_{2}-y_{2}\right)^{2}+\left(x_{3}-y_{3}\right)^{2}, \\
S^{2} & =\left(x_{2}-y_{2}\right)^{2}+\left(x_{3}+y_{3}\right)^{2}, \\
\beta & =\mu_{1} / \mu_{2}, \\
\alpha_{1} & =\left(\lambda_{1}+\mu_{1}\right) /\left(\lambda_{1}+2 \mu_{1}\right), \\
\alpha_{2} & =\left(\lambda_{2}+\mu_{2}\right) /\left(\lambda_{2}+2 \mu_{2}\right), \\
C_{1} & =2 \beta\left[\alpha_{2}\left(1-\beta-2 / \alpha_{1}\right)\right]^{-1}, \\
C_{2} & =(\beta-1)\left(1-\beta+2 \beta / \alpha_{2}\right)^{-1}, \\
D_{1} & =1+C_{2}, \\
D_{2} & =-\left(C_{1}+D_{1}\right) / 2 .
\end{aligned}
$$

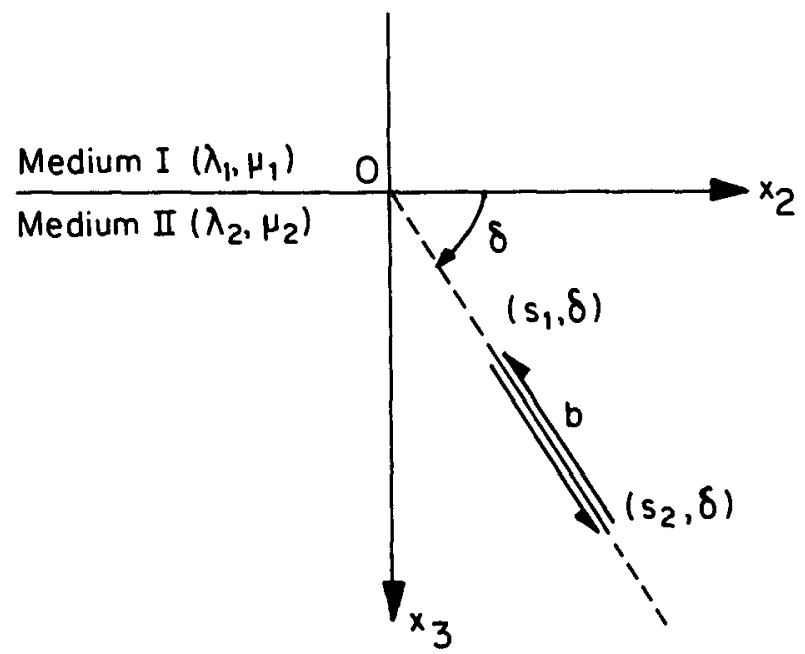

Figure 1. Two half-spaces in welded contact with a long dip-slip fault in the lower half-space. The Cartesian coordinates of a point on the fault are $\left(y_{2}, y_{3}\right)$ and its polar coordinates $(s, \delta)$, where $\delta$ is the dip angle and $s_{1} \leqslant s \leqslant s_{2}$.

We put (see figure 1)

$$
y_{2}=s \cos \delta, \quad y_{3}=s \sin \delta .
$$

Inserting the values of $y_{2}$ and $y_{3}$ from (7) into (4) and (5) and integrating over $s$ between the limits $\left(s_{1}, s_{2}\right)$, we obtain the following expressions for the Airy stress function for a long dip-slip fault of finite width $L=s_{2}-s_{1}$ :

$$
\begin{aligned}
U^{(1)}= & \left(\alpha_{2} \mu_{2} b / 2 \pi\right)\left[\left(C_{1}-D_{1}\right)\left(x_{3} \cos \delta-x_{2} \sin \delta\right)\{\ln R\}-C_{1} \sin 2 \delta\{s\}\right. \\
& +\left(C_{1}+D_{1}\right) \sin 2 \delta\{s \ln R\}+\left(C_{1}+D_{1}\right)\left(x_{3} \sin \delta+x_{2} \cos \delta\right)
\end{aligned}
$$




$$
\begin{aligned}
& \times\left\{\tan ^{-1}\left(\frac{s-x_{2} \cos \delta-x_{3} \sin \delta}{x_{2} \sin \delta-x_{3} \cos \delta}\right)\right\} \\
& \left.-\left(C_{1}+D_{1}\right) \cos 2 \delta\left\{s \tan ^{-1}\left(\frac{x_{2}-s \cos \delta}{x_{3}-s \sin \delta}\right)\right\}\right]\left.\right|_{s_{1}} ^{s_{2}} \\
U^{(2)}= & \left(\alpha_{2} \mu_{2} b / \pi\right)\left[\left(x_{2} \sin \delta-x_{3} \cos \delta\right)\{\ln R\}+\left(C_{2}+D_{2}\right)\left(x_{2} \sin \delta-x_{3} \cos \delta\right)\right. \\
& \times\{\ln S\}+\left(1+C_{2}+2 D_{2}\right) \sin \delta \cos \delta\{s\}-D_{2} \sin 2 \delta\{s \ln S\} \\
& -D_{2} \cos 2 \delta\left\{s \tan -1\left(\frac{x_{2}-s \cos \delta}{x_{3}+s \sin \delta}\right)\right\} \\
& -D_{2}\left(x_{2} \cos \delta+x_{3} \sin \delta\right)\left\{\tan -1\left(\frac{s+x_{3} \sin \delta-x_{2} \cos \delta}{x_{2} \sin \delta+x_{3} \cos \delta}\right)\right\} \\
& -\left.2 C_{2} x_{3} \sin \delta\left(x_{2} \sin \delta+x_{3} \cos \delta\right)\left\{\frac{s}{s^{2}}\right\}\right|_{s_{1}} ^{s_{2}}
\end{aligned}
$$

where now

$$
\begin{aligned}
& R^{2}=\left(x_{2}-s \cos \delta\right)^{2}+\left(x_{3}-s \sin \delta\right)^{2} \\
& S^{2}=\left(x_{2}-s \cos \delta\right)^{2}+\left(x_{3}+s \sin \delta\right)^{2}
\end{aligned}
$$

\section{Stresses and displacements}

From equations (2), (8) and (9), we get the following expressions for the stresses

$$
\begin{aligned}
p_{22}^{(1)}= & \left(\alpha_{2} \mu_{2} b / 2 \pi\right)\left[C_{1}\left(5 x_{3} \cos \delta-3 x_{2} \sin \delta\right)\left\{\frac{1}{R^{2}}\right\}\right. \\
& -D_{1}\left(x_{2} \sin \delta+x_{3} \cos \delta\right)\left\{\frac{1}{R^{2}}\right\}+2 D_{1} \sin 2 \delta\left\{\frac{s}{R^{2}}\right\} \\
& +4 C_{1} x_{3}\left(x_{2} \sin \delta-x_{3} \cos \delta\right)\left\{\frac{x_{3}-s \sin \delta}{R^{4}}\right\} \\
& \left.+4 D_{1} \sin \delta\left(x_{2} \sin \delta-x_{3} \cos \delta\right)\left\{\frac{s\left(x_{3}-s \sin \delta\right)}{R^{4}}\right\}\right]\left.\right|_{s_{1}} ^{s_{2}} \\
p_{23}^{(1)}= & \left(\alpha_{2} \mu_{2} b / 2 \pi\right)\left[\left(C_{1}-D_{1}\right)\left\{\frac{s}{R^{2}}\right\}-C_{1}\left(x_{2} \cos \delta+3 x_{3} \sin \delta\right)\left\{\frac{1}{R^{2}}\right\}\right. \\
& -D_{1}\left(x_{3} \sin \delta-x_{2} \cos \delta\right)\left\{\frac{1}{R^{2}}\right\}+4 C_{1} x_{3}\left(x_{2} \cos \delta+x_{3} \sin \delta\right) \\
& \times\left\{\frac{x_{3}-s \sin \delta}{R^{4}}\right\}+4\left[D_{1} \sin \delta\left(x_{2} \cos \delta+x_{3} \sin \delta\right)-C_{1} x_{3}\right] \\
& \times\left\{\frac{s\left(x_{3}-s \sin \delta\right)}{R^{4}}\right\}-\left.4 D_{1} \sin \delta\left\{\frac{s^{2}\left(x_{3}-s \sin \delta\right)}{R^{4}}\right\}\right|_{s_{1}} ^{s_{2}},
\end{aligned}
$$




$$
\begin{aligned}
& p_{33}^{(1)}=\left(\alpha_{2} \mu_{2} b / 2 \pi\right)\left[\left(D_{1}-C_{1}\right)\left(x_{2} \sin \delta+x_{3} \cos \delta\right)\left\{\frac{1}{R^{2}}\right\}\right. \\
& -2 D_{1} \sin 2 \delta\left\{\frac{s}{R^{2}}\right\}+4 C_{1} x_{3}\left(x_{3} \cos \delta-x_{2} \sin \delta\right)\left\{\frac{x_{3}-s \sin \delta}{R^{4}}\right\} \\
& \left.+4 D_{1} \sin \delta\left(x_{3} \cos \delta-x_{2} \sin \delta\right)\left\{\frac{s\left(x_{3}-s \sin \delta\right)}{R^{4}}\right\}\right]\left.\right|_{s_{1}} ^{s_{2}}, \\
& p_{22}^{(2)}=\left(\alpha_{2} \mu_{2} b / \pi\right)\left[\left(x_{2} \sin \delta-3 x_{3} \cos \delta\right)\left\{\frac{1}{R^{2}}\right\}+\sin 2 \delta\left\{\frac{s}{R^{2}}\right\}\right. \\
& -2\left(x_{2} \sin \delta-x_{3} \cos \delta\right)\left\{\frac{\left(x_{3}-s \sin \delta\right)^{2}}{R^{4}}\right\}-D_{2}\left(x_{2} \sin \delta+x_{3} \cos \delta\right)\left\{\frac{1}{S^{2}}\right\} \\
& +C_{2}\left(x_{2} \sin \delta-3 x_{3} \cos \delta\right)\left\{\frac{1}{S^{2}}\right\}-3 C_{2} \sin 2 \delta\left\{\frac{s}{S^{2}}\right\} \\
& -2 C_{2}\left(x_{2} \sin \delta-x_{3} \cos \delta\right)\left\{\frac{\left(x_{3}+s \sin \delta\right)^{2}}{S^{4}}\right\} \\
& +4 C_{2} x_{3} \sin \delta\left(3 x_{2} \sin \delta+5 x_{3} \cos \delta\right)\left\{\frac{s}{s^{4}}\right\} \\
& +8 C_{2} \sin ^{2} \delta\left(2 x_{3} \cos \delta+x_{2} \sin \delta\right)\left\{\frac{s^{2}}{S^{4}}\right\} \\
& \left.-16 C_{2} x_{3} \sin \delta\left(x_{2} \sin \delta+x_{3} \cos \delta\right)\left\{\frac{s\left(x_{3}+s \sin \delta\right)^{2}}{s^{6}}\right\}\right]\left.\right|_{s_{1}} ^{s_{2}} \\
& p_{23}^{(2)}=\left(\alpha_{2} \mu_{2} b / \pi\right)\left[\left(x_{2} \cos \delta+x_{3} \sin \delta\right)\left\{\frac{1}{R^{2}}+\frac{C_{2}}{S^{2}}\right\}-\left\{\frac{s}{R^{2}}\right\}\right. \\
& -2\left(x_{2} \cos \delta+x_{3} \sin \delta\right)\left\{\frac{\left(x_{3}-s \sin \delta\right)^{2}}{R^{4}}\right\}+2\left\{\frac{s\left(x_{3}-s \sin \delta\right)^{2}}{R^{4}}\right\} \\
& +D_{2}\left(x_{2} \cos \delta-x_{3} \sin \delta\right)\left\{\frac{1}{S^{2}}\right\}-\left(C_{2}+D_{2}\right)\left\{\frac{s}{S^{2}}\right\} \\
& -2 C_{2}\left(x_{2} \cos \delta+x_{3} \sin \delta\right)\left\{\frac{\left(x_{3}+s \sin \delta\right)^{2}}{s^{4}}\right\}+2 C_{2}\left\{\frac{s\left(x_{3}+s \sin \delta\right)^{2}}{s^{4}}\right\} \\
& -2 C_{2} x_{3} \sin 2 \delta\left\{\frac{s\left(x_{2}-s \cos \delta\right)}{s^{4}}\right\}+12 C_{2} x_{3} \sin ^{2} \delta\left\{\frac{s\left(x_{3}+s \sin \delta\right)}{s^{4}}\right\} \\
& +16 C_{2} x_{3} \sin \delta\left(x_{2} \cos \delta-x_{3} \sin \delta\right)\left\{\frac{s\left(x_{3}+s \sin \delta\right)^{2}}{s^{6}}\right\} \\
& \left.-16 C_{2} x_{3} \sin \delta\left\{\frac{s^{2}\left(x_{3}+s \sin \delta\right)^{2}}{s^{6}}\right\}\right]\left.\right|_{s_{1}} ^{s_{2}} \text {, }
\end{aligned}
$$




$$
\begin{aligned}
p_{33}^{(2)}= & \left(\alpha_{2} \mu_{2} b / \pi\right)\left[\left(x_{2} \sin \delta+x_{3} \cos \delta\right)\left\{\frac{1}{R^{2}}\right\}-\sin 2 \delta\left\{\frac{s}{R^{2}}\right\}\right. \\
& +2\left(x_{2} \sin \delta-x_{3} \cos \delta\right)\left\{\frac{\left(x_{3}-s \sin \delta\right)^{2}}{R^{4}}\right\}-C_{2} \sin 2 \delta\left\{\frac{s}{s^{2}}\right\} \\
& +\left(C_{2}+D_{2}\right)\left(x_{2} \sin \delta+x_{3} \cos \delta\right)\left\{\frac{1}{S^{2}}\right\} \\
& +2 C_{2}\left(x_{2} \sin \delta-x_{3} \cos \delta\right)\left\{\frac{\left(x_{3}+s \sin \delta\right)^{2}}{S^{4}}\right\} \\
& -4 C_{2} x_{3} \sin \delta\left(x_{2} \sin \delta+3 x_{3} \cos \delta\right)\left\{\frac{s}{s^{4}}\right\}-4 C_{2} x_{3} \sin \delta \sin 2 \delta\left\{\frac{s^{2}}{s^{4}}\right\} \\
& +\left.16 C_{2} x_{3} \sin \delta\left(x_{2} \sin \delta+x_{3} \cos \delta\right)\left\{\frac{s\left(x_{3}+\sin \delta\right)^{2}}{S^{6}}\right\}\right|_{s_{1}} ^{s_{2}}
\end{aligned}
$$

Corresponding to the stresses given by equations (11)-(16), the displacements are found by integrating the stress-strain relations Sokolnikoff 1956; Singh and Garg 1985):

$$
\begin{aligned}
& 2 \mu_{1} u_{2}^{(1)}=\left(\alpha_{2} \mu_{2} b / 2 \pi\right)\left[\left(C_{1}-D_{1}-2 C_{1} / \alpha_{1}\right) \sin \delta\{\ln R\}\right. \\
&-\left(C_{1}+D_{1}\right) \cos \delta\left\{\tan ^{-1}\left(\frac{s-x_{2} \cos \delta-x_{3} \sin \delta}{x_{2} \sin \delta-x_{3} \cos \delta}\right)\right\} \\
&+\left(2 C_{1} / \alpha_{1}\right) \cos \delta\left\{\tan ^{-1}\left(\frac{x_{2}-s \cos \delta}{x_{3}-s \sin \delta}\right)\right\} \\
&+\left.2\left\{\frac{\left(C_{1} x_{3}+D_{1} s \sin \delta\right)\left(s-x_{2} \cos \delta-x_{3} \sin \delta\right)}{R^{2}}\right\}\right|_{s_{1}} ^{s_{2}} \\
& 2 \mu_{1} u_{3}^{(1)}=\left(\alpha_{2} \mu_{2} b / 2 \pi\right)\left[\left(D_{1}-C_{1}+2 C_{1} / \alpha_{1}\right) \cos \delta\{\ln R\}\right. \\
&-\left(C_{1}+D_{1}\right) \sin \delta\left\{\tan -1\left(\frac{s-x_{2} \cos \delta-x_{3} \sin \delta}{x_{2} \sin \delta-x_{3} \cos \delta}\right)\right\} \\
&-\left(2 C_{1} / \alpha_{1}\right) \sin \delta\left\{\tan -1\left(\frac{x_{3}-s \sin \delta}{x_{2}-s \cos \delta}\right)\right\} \\
&+\left.2\left(x_{2} \sin \delta-x_{3} \cos \delta\right)\left\{\frac{C_{1} x_{3}+D_{1} s \sin \delta}{R^{2}}\right\}\right|_{s_{1}} ^{s_{2}} \\
&-\left(\cos \delta / \alpha_{2}\right)\left\{\tan -1\left(\frac{x_{2}-s \cos \delta}{x_{3}-s \sin \delta}\right)\right\} \\
& 2 \mu_{2} u_{2}^{(2)}=\left(\alpha_{2} \mu_{2} b / \pi\right)\left[-\left(1-1 / \alpha_{2}\right) \sin \delta\{\ln R\}-\left(C_{2}+D_{2}-C_{2} / \alpha_{2}\right) \sin \delta\{\ln S\}\right. \\
&-18) \\
& \\
&
\end{aligned}
$$




$$
\begin{aligned}
& -\left(C_{2} / \alpha_{2}\right) \cos \delta\left\{\tan ^{-1}\left(\frac{x_{2}-s \cos \delta}{x_{3}+s \sin \delta}\right)\right\} \\
& +D_{2} \cos \delta\left\{\tan ^{-1}\left(\frac{s+x_{3} \sin \delta-x_{2} \cos \delta}{x_{2} \sin \delta+x_{3} \cos \delta}\right)\right\} \\
& +\left\{\left(x_{3}-s \sin \delta\right)\left(x_{2} \cos \delta+x_{3} \sin \delta-s\right)\left(\frac{1}{R^{2}}+\frac{C_{2}}{S^{2}}\right)\right\} \\
& +2 C_{2} x_{3} \sin ^{2} \delta\left\{\frac{s}{s^{2}}\right\}+\left(2 / \alpha_{2}\right) C_{2} \sin \delta\left(x_{2} \cos \delta-x_{3} \sin \delta\right)\left\{\frac{s}{s^{2}}\right\} \\
& -\left(2 / \alpha_{2}\right) C_{2} \sin \delta\left\{\frac{s^{2}}{s^{2}}\right\}+4 C_{2} x_{3} \sin \delta \\
& \left.\times\left\{\frac{s\left(x_{3}+s \sin \delta\right)\left(\dot{x_{3}} \sin \delta-x_{2} \cos \delta+s\right)}{s^{4}}\right\}\right]\left.\right|_{s_{1}} ^{s_{2}} \text {, } \\
& 2 \mu_{2} u_{3}^{(2)}=\left(\alpha_{2} \mu_{2} b / \pi\right)\left[\left(1-1 / \alpha_{2}\right) \cos \delta\{\ln R\}+\left(D_{2}+C_{2}-C_{2} / \alpha_{2}\right) \cos \delta\{\ln S\}\right. \\
& +\left(\sin \delta / \alpha_{2}\right)\left\{\tan ^{-1}\left(\frac{x_{3}-s \sin \delta}{x_{2}-s \cos \delta}\right)\right\} \\
& +\left(C_{2} \sin \delta / \alpha_{2}\right)\left\{\tan ^{-1}\left(\frac{x_{3}+s \sin \delta}{x_{2}-s \cos \delta}\right)\right\} \\
& +D_{2} \sin \delta\left\{\tan ^{-1}\left(\frac{s+x_{3} \sin \delta-x_{2} \cos \delta}{x_{2} \sin \delta+x_{3} \cos \delta}\right)\right\} \\
& -\left(x_{2} \sin \delta-x_{3} \cos \delta\right)\left\{\frac{x_{3}-s \sin \delta}{R^{2}}+\frac{C_{2}\left(x_{3}+s \sin \delta\right)}{S^{2}}\right\} \\
& +2 C_{2} \sin \delta\left(x_{2} \sin \delta+2 x_{3} \cos \delta\right)\left\{\frac{s}{S^{2}}\right\} \\
& -\left(2 / \alpha_{2}\right) C_{2} \sin \delta\left(x_{2} \sin \delta+x_{3} \cos \delta\right)\left\{\frac{s}{s^{2}}\right\} \\
& \left.-4 C_{2} x_{3} \sin \delta\left(x_{2} \sin \delta+x_{3} \cos \delta\right)\left\{\frac{s\left(x_{3}+s \sin \delta\right)}{s^{4}}\right\}\right]\left.\right|_{s_{1}} ^{s_{2}}
\end{aligned}
$$

We have verified that the stresses and the displacements given in equations (12)-(20) satisfy the necessary continuity conditions

$$
p_{23}^{(1)}=p_{23}^{(2)}, \quad p_{33}^{(1)}=p_{33}^{(2)}, \quad u_{2}^{(1)}=u_{2}^{(2)}, \quad u_{3}^{(1)}=u_{3}^{(2)}
$$

at $x_{3}=0$.

The results for the displacements and stresses in a uniform half-space can be obtained as a particular case on putting $\mu_{1}=0$. From equation (6), we note that this implies $\beta=0, C_{2}=-1, C_{1}=D_{1}=D_{2}=0$. It has been verified that the results obtained on putting $\mu_{1}=0$ in equations (14)-(16), (19) and (20) coincide with the corresponding results of Rani and Singh (1992) for a uniform half-space. 


\section{Numerical results}

For numerical computations we assume Poissonian (i.e. $\lambda_{i}=\mu_{i}$ ) half-spaces so that

$$
\begin{aligned}
& \alpha_{1}=\alpha_{2}=2 / 3, \\
& C_{1}=-3 \beta /(2+\beta), \\
& D_{1}=3 \beta /(1+2 \beta) .
\end{aligned}
$$

We define the following dimensionless quantities

$$
\begin{array}{ll}
Y=x_{2} / L, & Z=x_{3} / L, \\
U_{i}^{(1)}=u_{i}^{(1)} / b, & U_{i}^{(2)}=u_{i}^{(2)} / b, \\
P_{i j}^{(1)}=\left(L / \mu_{2} b\right) p_{i j}^{(1)}, & P_{i j}^{(2)}=\left(L / \mu_{2} b\right) p_{i j}^{(2)},
\end{array}
$$

where $b$ is the magnitude of the dislocation (slip). Thus, $Y$ is the dimensionless distance (horizontal) from the fault, $Z$ is the dimensionless distance from the interface $(Z<0$ for medium I and $Z>0$ for medium II), $U_{2}$ and $U_{3}$ are, respectively, the dimensionless horizontal (parallel to the fault) and vertical (down) displacements and $P_{22}, P_{23}, P_{33}$ are dimensionless stresses.

We study numerically the displacement field at the interface $\left(x_{3}=0\right)$ for a long dip angle $\delta$ for $\mu_{1} / \mu_{2}=1 / 2$. From these figures we note that the displacement field and 3 show the variation of the dimensionless horizontal $\left(U_{2}\right)$ and vertical $\left(U_{3}\right)$ displacements at the interface with the distance from the fault for three values of the dip angle $\delta$ for $\mu_{1} / \mu_{2}=1 / 2$. From these figures we note that the displacement field changes significantly with the change in the dip angle. For $\delta=90^{\circ}, U_{2}$ is symmetric

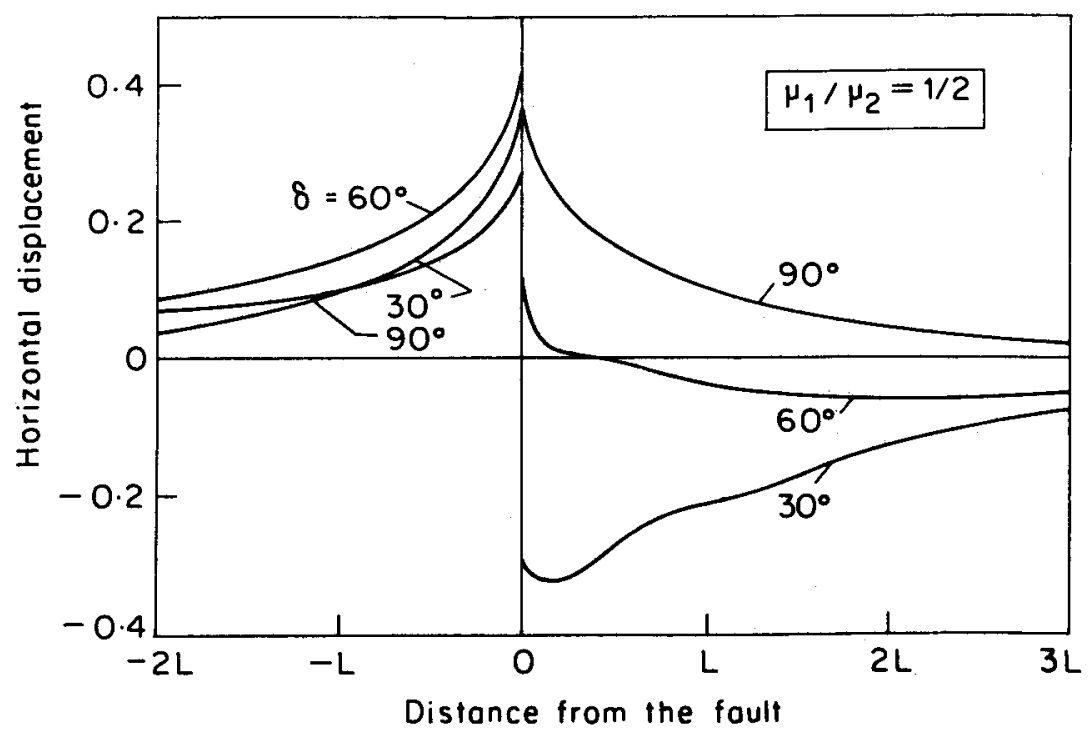

Figure 2. Variation of the horizontal displacement $\left(U_{2}\right)$ at the interface with the distance from the fault for $\mu_{1} / \mu_{2}=1 / 2$ for three values of the dip angle $\delta: 30^{\circ}, 60^{\circ}$ and $90^{\circ}$. 


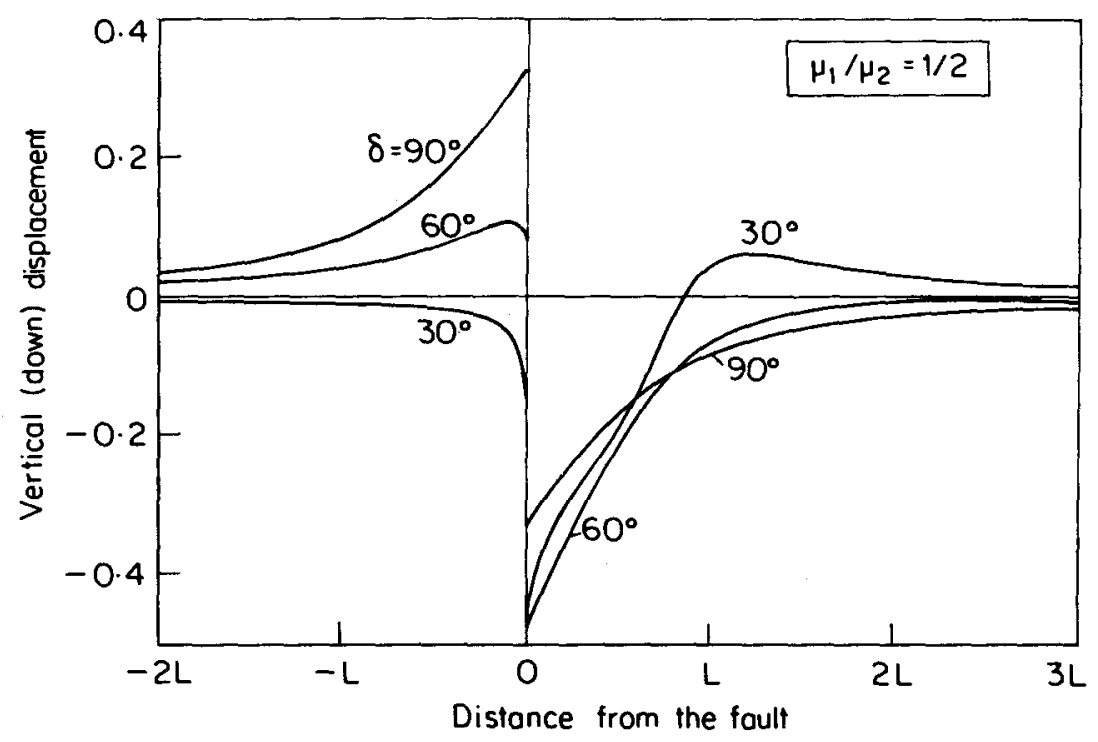

Figure 3. Variation of the vertical displacement $\left(U_{3}\right.$, positive downward) at the interface with the distance from the fault for $\mu_{1} / \mu_{2}=1 / 2$ at dip angles $30^{\circ}, 60^{\circ}$ and $90^{\circ}$.

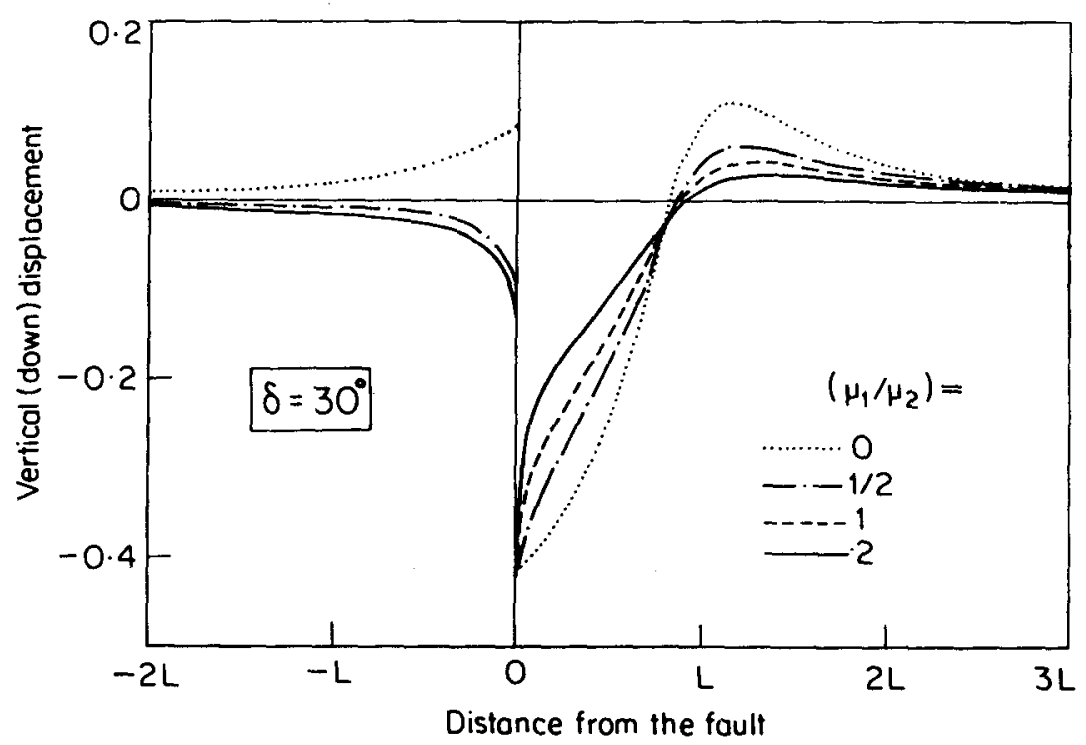

Figure 4. Variation of the vertical displacement $\left(U_{3}\right)$ at the interface with the distance from the fault for $\delta=30^{\circ}$. The curve $\mu_{1} / \mu_{2}=0$ is for a uniform half-space and the curve $\mu_{1} / \mu_{2}=1$ is for a uniform unbounded medium.

while $U_{3}$ is antisymmetric about the origin. For dip angles other than $90^{\circ}$, the displacement field is asymmetric, the degree of asymmetry depending upon the dip angle. Figures 4 and 5 display the variation of the vertical displacement $\left(U_{3}\right)$ at the interface for $\delta=30^{\circ}$ and $60^{\circ}$, respectively, for four values of the ratio of the rigidities 


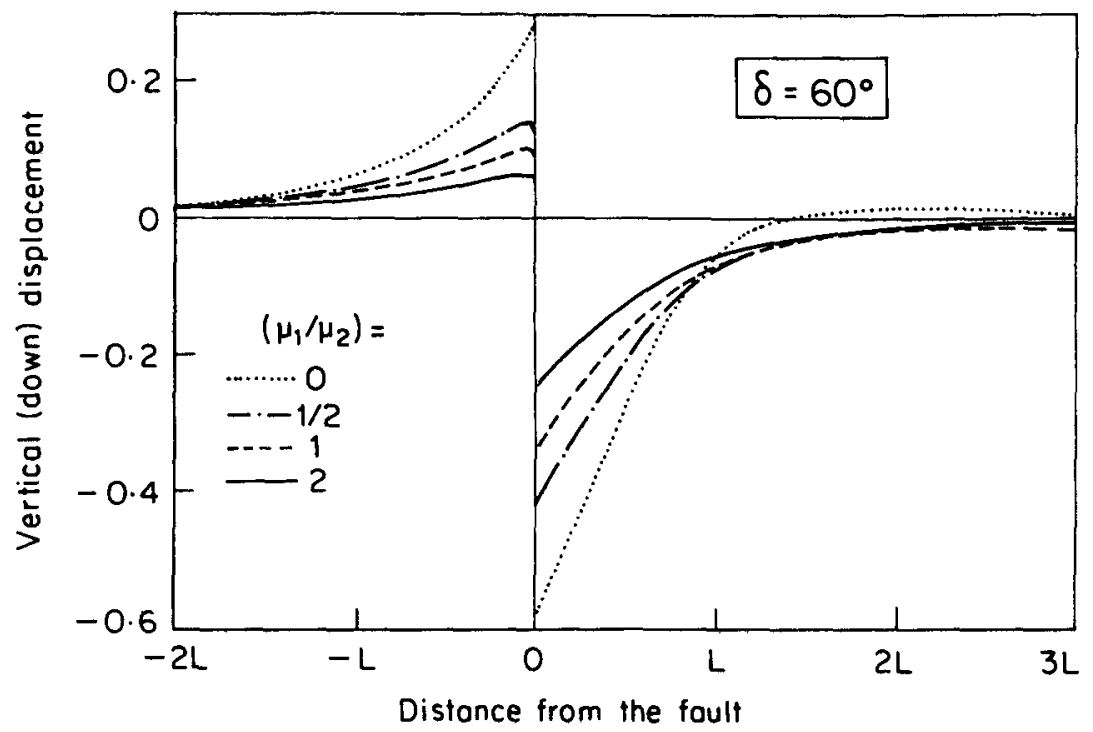

Figure 5. Variation of $U_{3}$ at the interface with the distance from the fault for $\delta=60^{\circ}$.

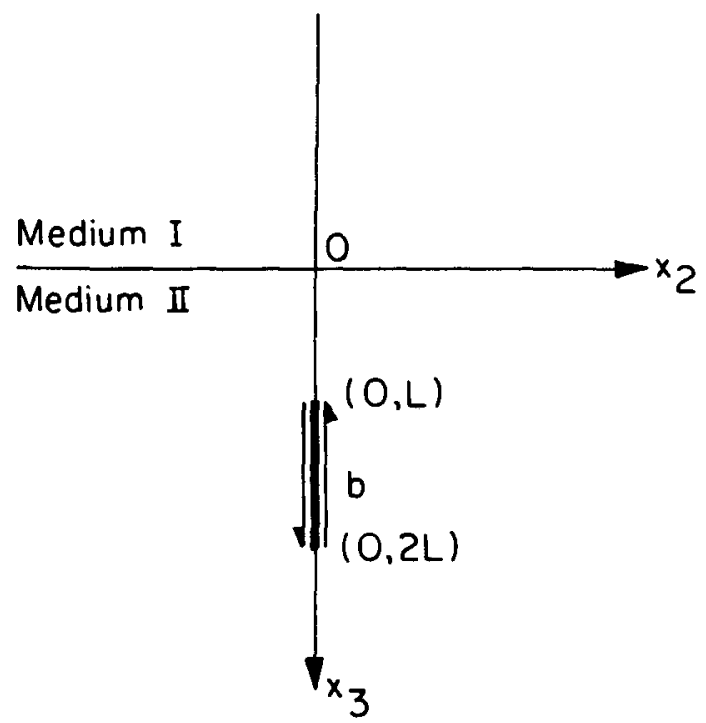

Figure 6. Geometry of a long vertical dip-slip fault occupying the region $x_{2}=0, L \leqslant x_{3} \leqslant 2 L$ of medium II.

of the two half-spaces, namely, $\mu_{1} / \mu_{2}=0,1 / 2,1$ and 2 . The case $\mu_{1} / \mu_{2}=0$ corresponds to a uniform half-space and the case $\mu_{1} / \mu_{2}=1$ to a uniform unbounded medium.

We next consider a long vertical dip-slip fault of width $L$, assuming $\delta=\pi / 2, s_{1}=L$, $s_{2}=2 L$ (figure 6). Figure 7 shows the variation of the dimensionless horizontal displacement $\left(U_{2}\right)$ with the distance from the interface at two epicentral locations, $y=L / 10, L$, for $\mu_{1} / \mu_{2}=1 / 2$. Similarly, figure 8 shows the variation of the vertical 


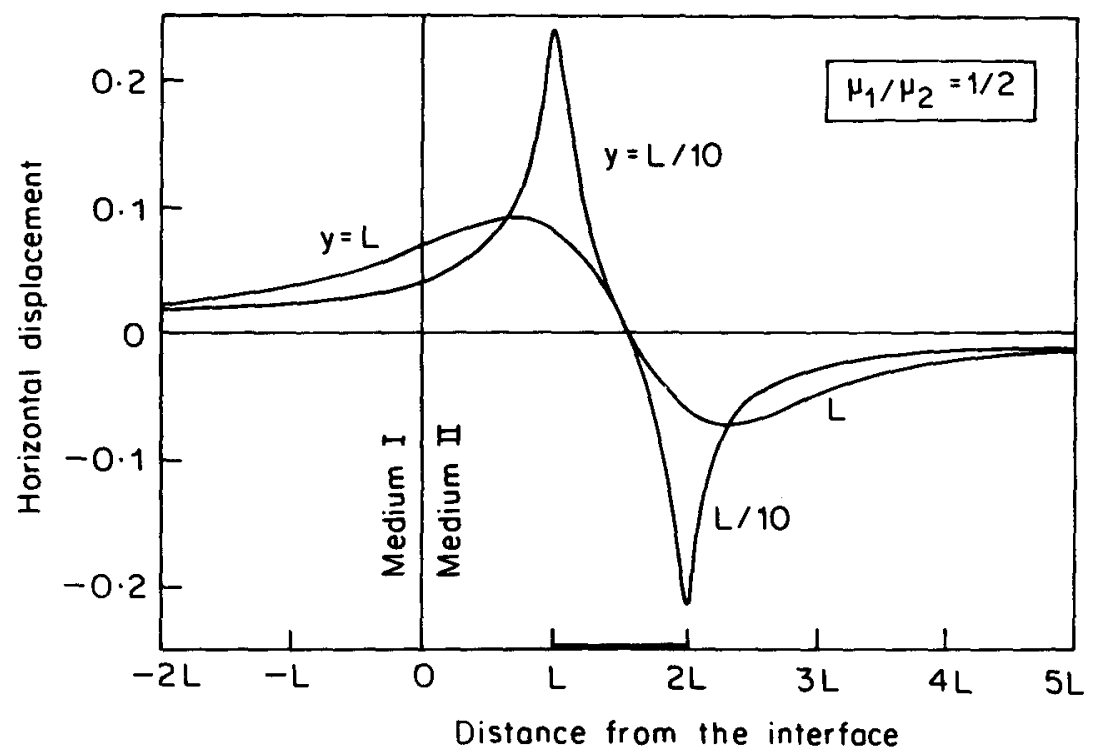

Figure 7. Variation of $U_{2}$ with the distance from the interface $\left(x_{3}\right)$ for $\mu_{1} / \mu_{2}=1 / 2$ at two epicentral locations: $y=L / 10, L$. Negative values of $x_{3}$ refer to medium I, positive values refer to medium II.

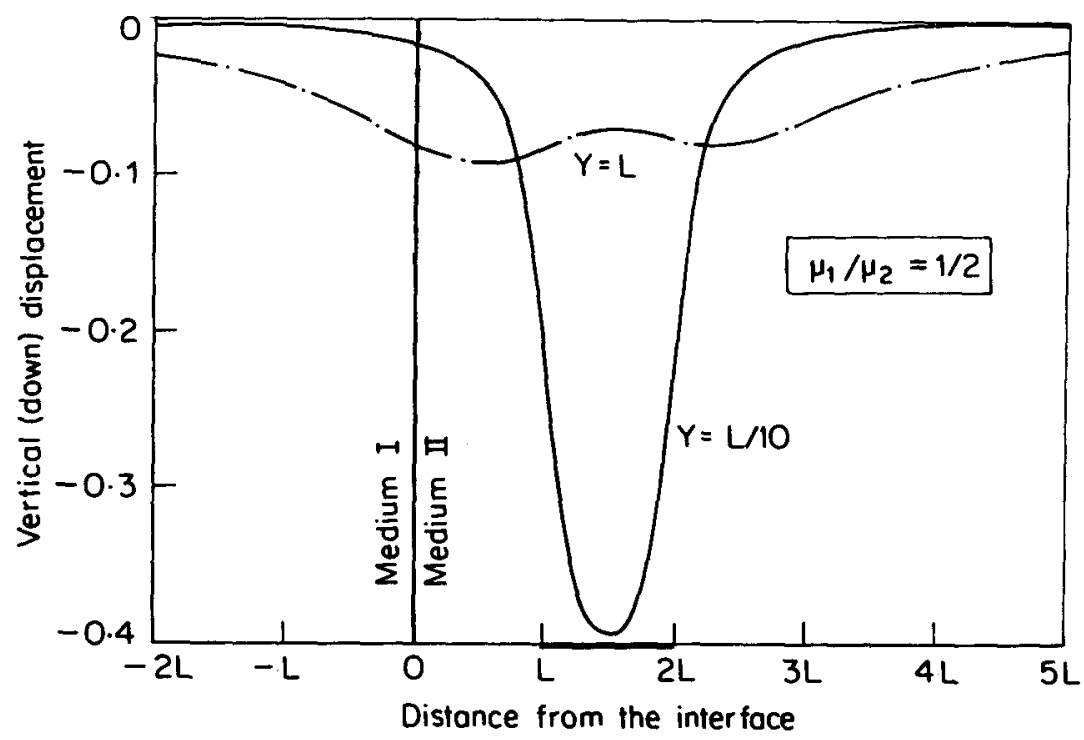

Figure 8. Variation of $U_{3}$ with the distance from the interface $\left(x_{3}\right)$ for $\mu_{1} / \mu_{2}=1 / 2$ at two epicentral locations: $y=L / 10, L$.

displacement $\left(U_{3}\right)$ with the distance from the interface. From these figures we note that the variation of the horizontal displacement is significant in the range $0<z<3 L$, especially near the fault $(y=L / 10)$. Similarly, the vertical displacement varies significantly in the range $0<z<3 L$ for $y=L / 10$. While the horizontal displacement 


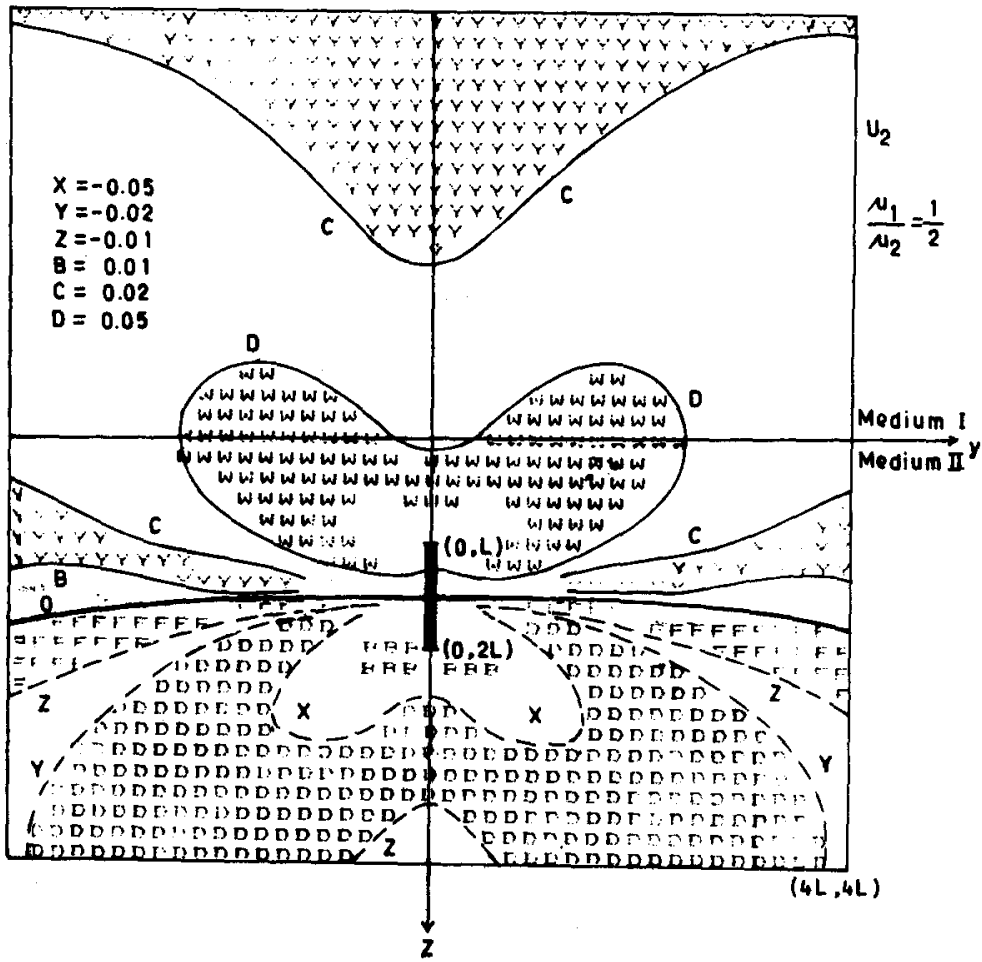

Figure 9. Contour map for $U_{2}$ for $\mu_{1}^{\prime} \mu_{2}=1 / 2$. The contour values of isolines have been indicated. Solid lines correspond to positive values, broken lines correspond to negative values. Heavy line indicates fault trace.

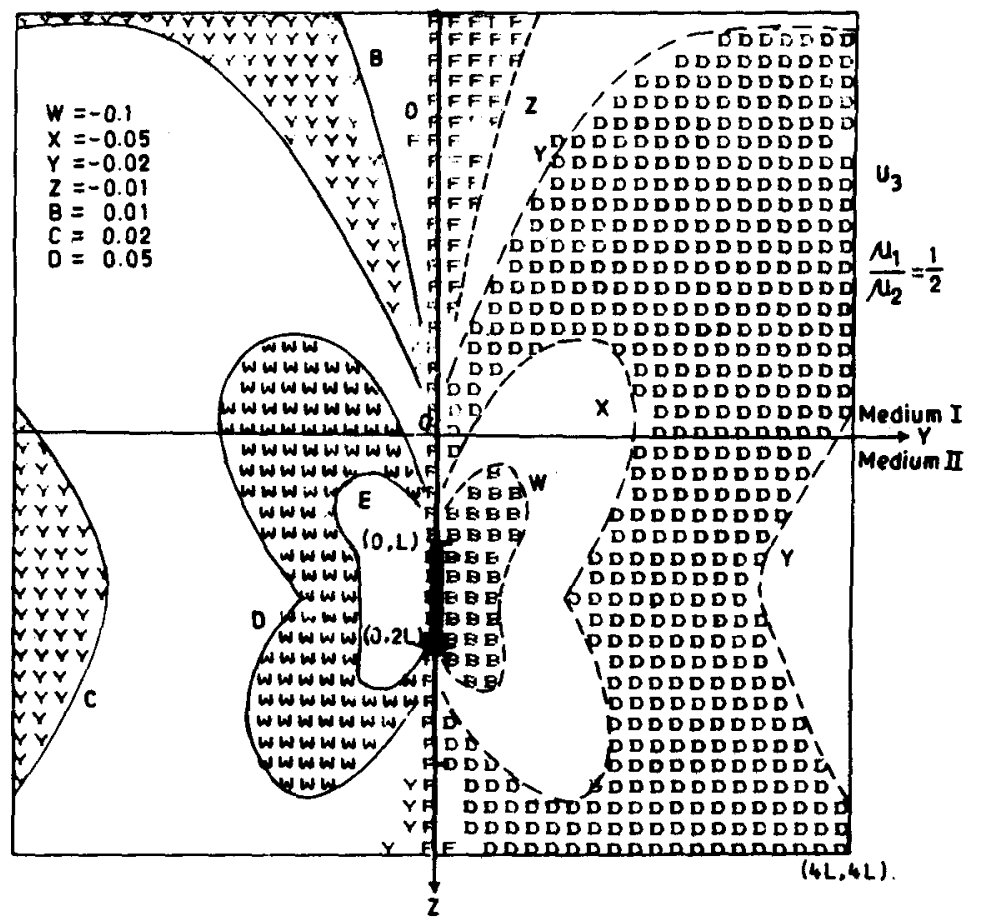

Figure 10. Contour map for $U_{3}$ for $\mu_{1} / \mu_{2}=1 / 2$. 


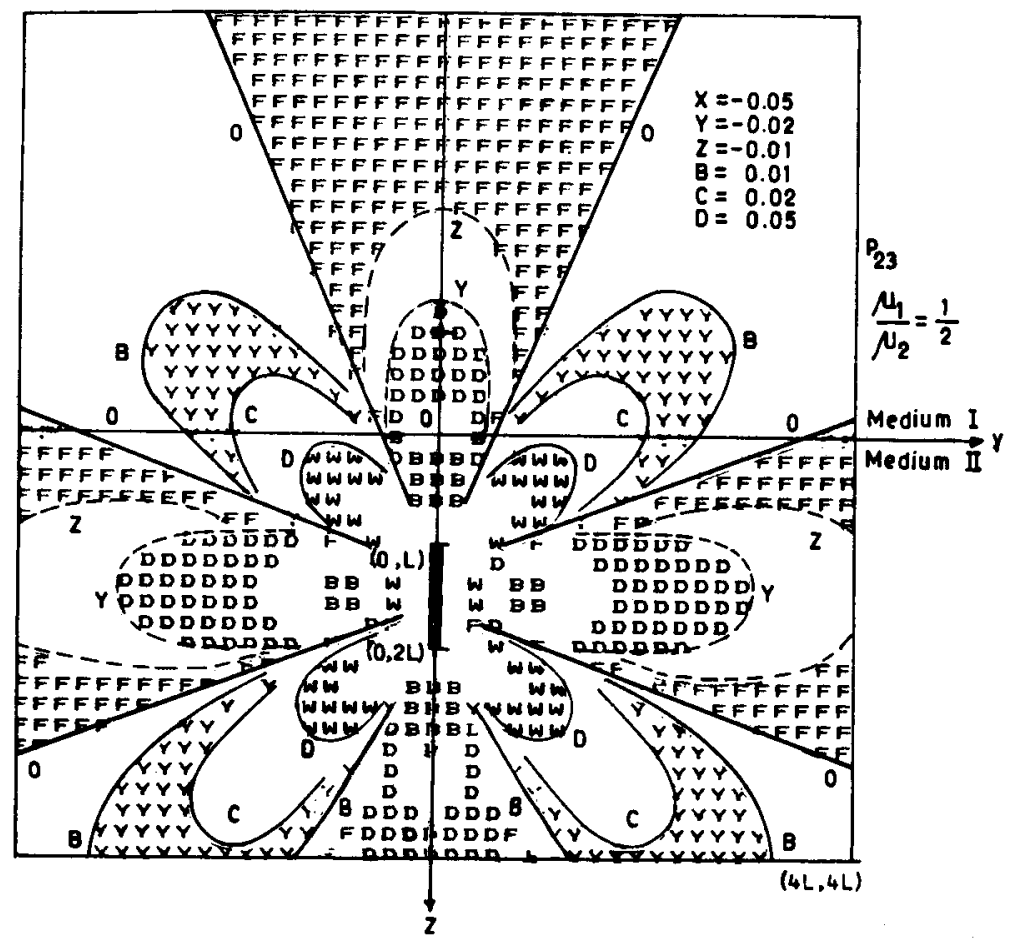

Figure 11. Contour map for the shear stress $\left(P_{23}\right)$ for $\mu_{1} / \mu_{2}=1 / 2$.

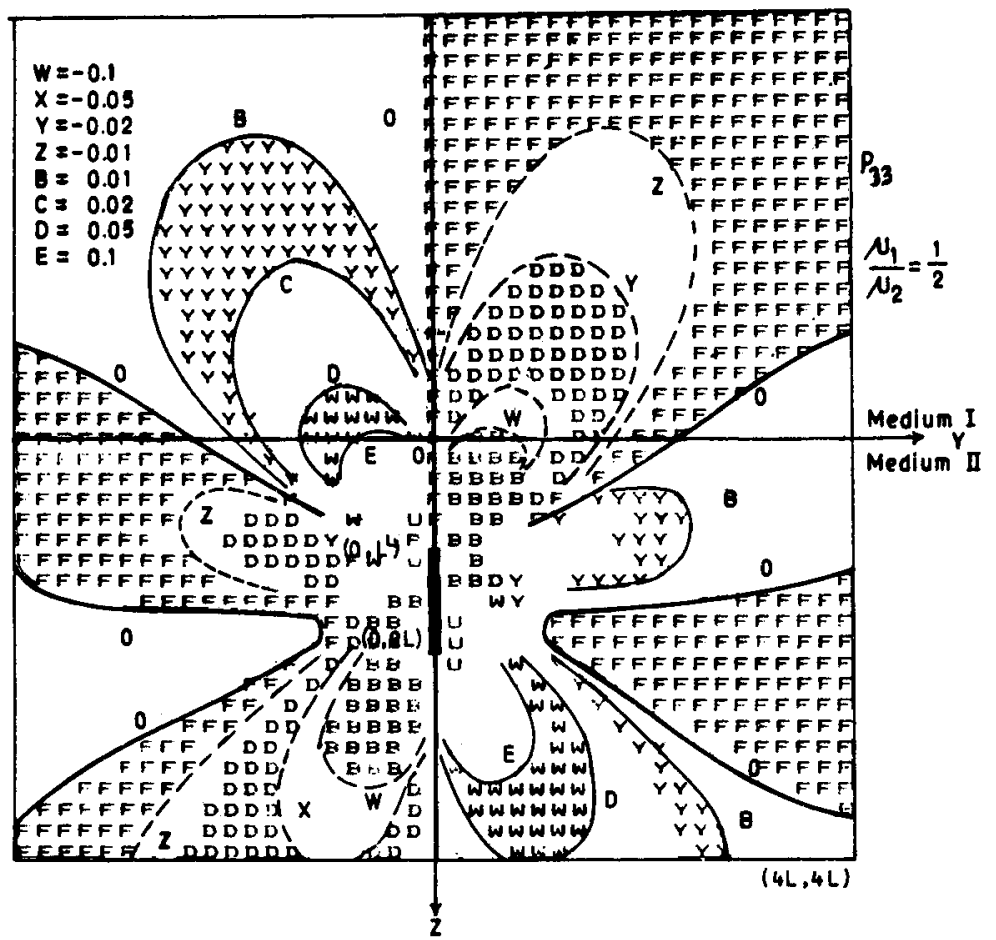

Figure 12. Contour map for the normal stress $\left(P_{33}\right)$ for $\mu_{1} / \mu_{2}=1 / 2$. 
is maximum near the fault ends, the vertical displacement is maximum near the middle of the fault.

Contour maps in vertical planes perpendicular to the length of the fault for the displacements $U_{2}, U_{3}$ and stresses $P_{23}, P_{33}$ are given in figures 9 to 12 for $\mu_{1} / \mu_{2}=1 / 2$. The contour values for the isolines are indicated. Solid lines denote positive values and broken lines denote negative values. Nodal lines are also drawn. These maps exhibit the variation of the elastic field around the fault in the two media.

\section{Acknowledgements}

The authors are thankful to the University Grants Commission, New Delhi for financial support and to the anonymous referees for their useful comments which led to an improvement in the presentation of the paper.

\section{References}

Chinnery M A and Jovanovich D B 1972 Effect of earth layering on earthquake displacement fields; Bull. Seismol. Soc. Am. 62 1629-1639

Freund L, B and Barnett D M 1976 A two-dimensional analysis of surface deformation due to dip-slip faulting; Bull. Seismol. Soc. Am. 66 667-675

Rani S and Singh S J 1992 Static deformation of a uniform half-space due to a long dip-slip fault; Geophys. J. Int. 109 469-476

Rani S, Singh S J and Garg N R 1991 Displacements and stresses at any point of a uniform half-space due to two-dimensional buried sources; Phys. Earth Planet. Int. 65 276-282

Rybicki K 1971 The elastic residual field of a very long strike-slip fault in the presence of discontinuity; Bull. Seismol. Soc. Am. 61 79-92

Rybicki K 1973 Static deformation of a multilayered half-space by a very long strike-slip fault; Pure Appl. Geophys. 110 1955-1966

Singh K and Singh S J 1990 A simple procedure for obtaining the quasi-static displacements, strains and stresses in a viscoelastic half-space; Bull. Seismol. Soc. Am. $80488-492$

Singh S J and Garg N R 1985 On two-dimensional elastic dislocations in a multilayered half-space; Phys. Earth Planet. Int. 40 135-145

Singh S J, Rani S and Garg N R 1992 Displacements and stresses in two welded half-spaces due to twodimensional sources; Phys. Earth. Planet. Int. 70 90-101

Singh S J and Rosenman M-1974 Quasi-static deformation of a viscoelastic half-space by shear dislocations; Phys. Earth Planet. Int. 8 87-101

Sokolnikoff I S 1956 Mathematical Theory of Elasticity (New York: McGraw-Hill) 